\title{
LOW- AND INTERMEDIATE-VELOCITY HYDROGEN AT POSITIVE GALACTIC LATITUDES
}

\author{
K. TAKAKUBO \\ Astronomical Institute, Tohoku University, Sandai, Japan
}

\begin{abstract}
Low- and intermediate-velocity Gaussian components of hydrogen emission profiles observed at positive galactic latitudes are investigated. The low-velocity hole, as called by Wesselius and Fejes (1973), is a phenomenon which appears in the layer composed of the gas emitting narrow Gaussian components but does not in that of wide components. Both Models I and II suggested by Wesselius and Fejes contradict this result. A hypothesis is proposed that a stream motion in the layer emitting wide Gaussian components, which may be identified with the intercloud medium, swept away the interstellar clouds emitting narrow Gaussian components in the region of the hole.
\end{abstract}

A remarkable deficiency of low-velocity hydrogen gas at high positive latitudes has been shown by several investigators. Among them, Wesselius and Fejes (1973) called it the low-velocity hole and showed that there was a good correspondence in position and shape between the hole and a negative-velocity hydrogen complex.

In the present paper, we will consider low- and intermediate-velocity gas at positive latitudes and point out that the hole appears only in the layer of the gas emitting narrow Gaussian components but not in one emitting wide Gaussian components.

The data are 309 hydrogen emission profiles selected from observations by Tolbert (1971) at high galactic latitudes and by Takakubo and van Woerden (1966) at intermediate latitudes. These observed points more or less uniformly cover the northern hemisphere at latitudes higher than $10^{\circ}\left(\Delta b \sim 5^{\circ}, \Delta l \cdot \cos b \sim 10^{\circ}\right)$.

Each profile observed is transformed into optical depth, $\tau(V)$ as a function of the radial velocity, $V$, with respect to the local standard of rest, assuming a uniform temperature of $125 \mathrm{~K}$. Then it is expressed by a superposition of Gaussian components $\left(\tau_{\mathrm{i}}, V_{\mathrm{i}}, \sigma_{\mathrm{i}}\right)$ :

$$
\tau(V)=\sum_{\mathrm{i}} \tau_{\mathrm{i}} \exp \left\{-\left(V-V_{\mathrm{i}}\right)^{2} / 2 \sigma_{\mathrm{i}}^{2}\right\} .
$$

The column density, $N_{\mathrm{Hi}}$, of a component is proportional to the product $\tau_{\mathrm{i}} \sigma_{\mathrm{i}}$ and does not depend on assumed temperature so much if the layer is optically thin.

Among these Gaussian components 1041 have radial velocities $V_{\mathrm{i}}$ between $-60 \mathrm{~km}$ $\mathrm{s}^{-1}$ and $+60 \mathrm{~km} \mathrm{~s}^{-1}$. We will use them as low- and intermediate-velocity components.

As pointed out in a previous paper (Takakubo, 1967), Gaussian components have rather different natures according to the values of their radial-velocity dispersion, $\sigma_{\mathrm{i}}(=0.85$ half half-width $)$. We will, thus, divide them into two classes,

$$
\begin{array}{ll}
\text { narrow components: } & \sigma_{\mathrm{i}} \leqq \sigma_{\text {bound }}, \\
\text { wide components: } & \sigma_{\mathrm{i}}>\sigma_{\text {bound }},
\end{array}
$$




\section{$N_{H} \cdot \sin b \quad$ NARROW COMPONENTS $\quad|\mathrm{V}| \leqq 60$ \\ b $>0$}

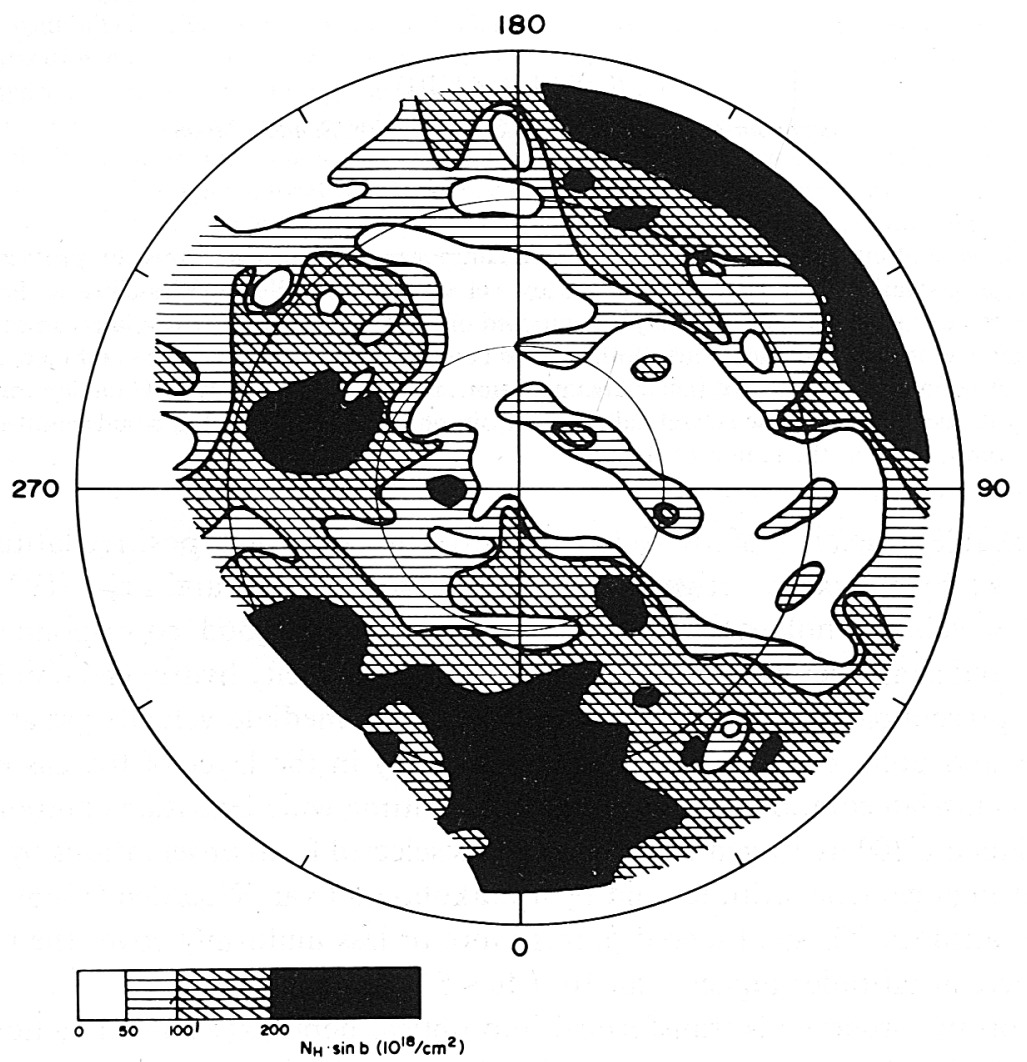

Fig. 1. The contour map of $\sum N_{\mathrm{Hi}} \cdot \sin b$ for narrow components $\left(\sigma_{\mathrm{i}} \leqq 7.0 \mathrm{~km} \mathrm{~s}^{-1}\right)$. The north galactic pole is at the centre; the three circles indicate $b=60^{\circ}, 30^{\circ}$, and $0^{\circ}$, respectively. Note that the low-velocity hole extends to considerably low latitude. The contour lines of this kind of figure cannot always be determined uniquely.

with

$$
\sigma_{\text {bound }}=7.0 \mathrm{~km} \mathrm{~s}^{-1} \text {. }
$$

The narrow components are of groups $S$ and $M$ in the previous paper, and the wide components are of group $L$. Although the boundary value $\sigma_{\text {bound }}=7.0 \mathrm{~km} \mathrm{~s}^{-1}$ was adopted more or less arbitrarily, it is a rather reasonable value (see Takakubo, 1967). The statistical investigation by Mebold (1972) also supports it, although he adopted a slightly smaller value of $\sigma_{\text {bound }}$. The classification in the present paper is essentially the same as his, and we may consider in a crude treatment that the narrow components are mainly due to the emission of the interstellar clouds and the wide ones to that of the intercloud medium. 


\section{$N_{H} \cdot \sin b \quad$ WIDE COMPONENTS $\quad|V| \leqq 60$ \\ $b>0$}

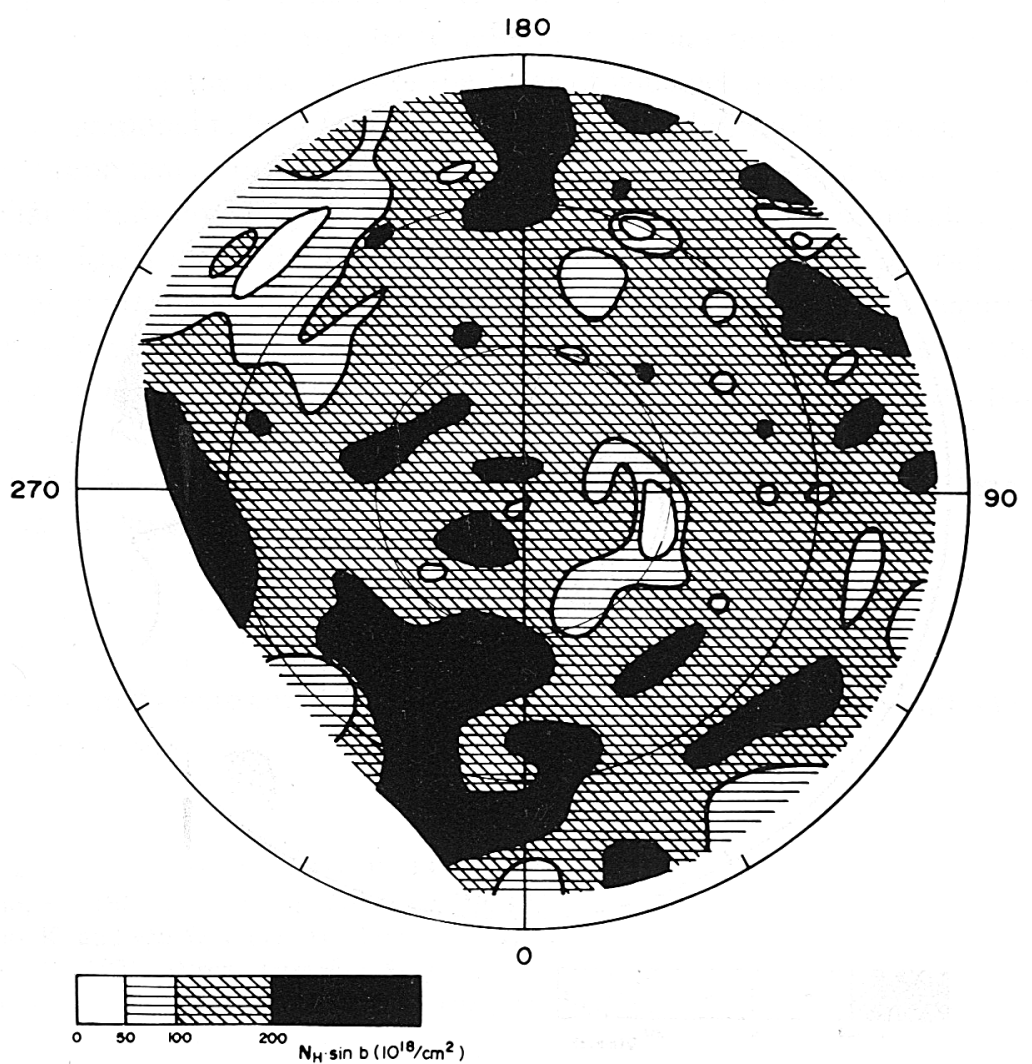

Fig. 2. The contour map of $\sum N_{\mathrm{Hi}} \cdot \sin b$ for wide components $\left(\sigma_{\mathrm{i}}>7.0 \mathrm{~km} \mathrm{~s}^{-1}\right)$.

At each point $(l, b)$

$$
\sum_{\mathbf{i}} N_{\mathrm{Hi}} \cdot \sin b
$$

is computed, where the summation extends over all the components in a profile. If the hydrogen gas is stratified parallel to the galactic plane, this quantity should be constant over the sky. But it is not the case, as is expected from previous investigations. Now we treat the narrow- and the wide-component classes separately and see the distribution of the quantity over the sky.

In the class of narrow components $\sum N_{\mathrm{Hi}} \cdot \sin b$ is not constant at all and has marked deviations from the plane parallel stratification (Figure 1). Patterns due to the low velocity hole, the tilted disk ('Scheve Schijf') and the Perseus Arm are conspicuous. Particularly, the low-velocity hole extends to much lower latitudes than Wesselius and Fejes (1973) suggested. 


\section{$\bar{v} \quad$ WIDE COMPONENTS $\quad|V| \leqq 60$ \\ $b>0$}

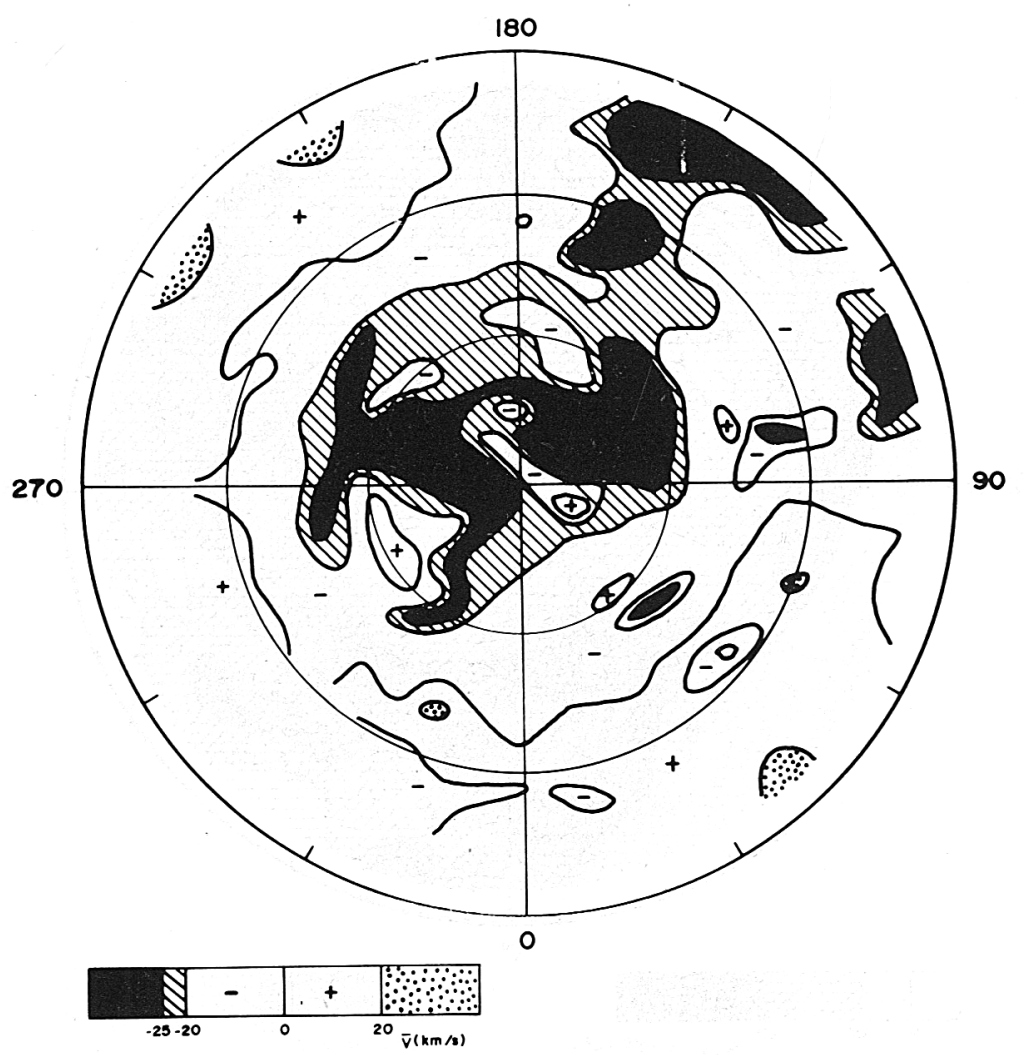

Fig. 3. The contour map of the average radial velocity $\bar{V}_{\mathrm{i}}$ for wide components.

On the other hand, in the class of wide components the distribution of $\sum N_{\mathrm{Hi}} \cdot \sin b$ is nearly uniform (Figure 2). Only in a few small regions does it deviate from the average, and no outstanding patterns appear, except one due to the tilted disk.

The difference in the distribution of $\sum N_{\mathrm{Hi}} \cdot \sin b$ of the two classes is also clear from the frequency distribution of $\sum N_{\mathrm{Hi}} \cdot \sin b$. We may conclude that the gas emitting wide Gaussian components is stratified parallel to the galactic plane and its column density perpendicular to the plane is $1.4 \times 10^{20} \mathrm{~cm}^{-2}$.

It is concluded, therefore, that the hole is a phenomenon in the layer of narrow Gaussian components, but it is absent in that of wide components. If the hole is due to either a nearby supernova explosion (Model I of Wesselius and Fejes, 1973) or a colliding big gas complex (Model II of theirs), the absence of the hole in the intercloud medium is hardly understandable, since in these models the hole is expected also in the intercloud medium. A hypothesis that the internal motion of interstellar 
clouds in the region of the hole is increased and the emission becomes broader by some reason meets a difficulty, since, if this is the case, the value of $\sum N_{\mathrm{Hi}} \cdot \sin b$ of wide components should be increased in the hole region by a factor of 2 or so.

A possible explanation may be to assume a stream motion in the layer of wide components. Since the thickness of the layer of wide components is larger than that of narrow components by a factor of 1.5 (see Takakubo, 1967), a stream in the layer of wide components originating high above the galactic plane does not contain the interstellar clouds emitting narrow components. If the stream penetrates into lower layers, the clouds along its passage will be blown away. Indeed, the radial velocity of wide components (Figure 3) shows marked negative deviation in the region to lower latitudes at around a galactic longitude of $150^{\circ}$; it is natural to assume the stream comes from this longitude direction. It is noticed that we find a high density part, called the tilted disk, just in the opposite direction.

It is not yet clear, however, if the stream hypothesis is compatible with the continuity of mass in the layer of wide components.

\section{Acknowledgement}

The computations were carried out by using a NEAC-2200 at the Computer Center at Tohoku University.

\section{References}

Mebold, U.: 1972, Astron. Astrophys. 19, 13.

Takakubo, K.: 1967, Bull. Astron. Inst. Neth. 19, 125.

Takakubo, K. and van Woerden, H.: 1966, Bull. Astron. Inst. Neth. 18, 488.

Tolbert, C. R.: 1971, Astron. Astrophys. Suppl. 3, 349.

Wesselius, P. R. and Fejes, I.: 1973, Astron. Astrophys. 24, 15.

\section{K. Takakubo}

Astronomical Institute,

Tohoku University,

Sendai, Japan 